\title{
Hydrogels utilizing G-quadruplexes
}

\begin{abstract}
Among recently reported unique and interesting hydrogel systems utilizing DNA as a component, those employing G-quadruplexes are particularly emerging. Their design principles and achieved functions are briefly reviewed.
\end{abstract}

Keywords: hydrogels, DNA, G-quartet, G-quadruplexes, injectable polymers, DDS, biodegradable polymers
Volume I Issue $6-2017$

\author{
Akinori Kuzuya, ',2 Shizuma Tanaka' \\ 'Department of Chemistry and Materials Engineering, Kansai \\ University, Japan \\ ${ }^{2}$ Collaborative Research Center of Engineering, Medicine and \\ Pharmacology, ORDIST, Kansai University, Japan
}

\begin{abstract}
Correspondence: Akinori Kuzuya, Department of Chemistry and Materials Engineering, Kansai University, 3-3-35 Yamate, Suita, Osaka 564-8680, Japan, Tel +8I-6-6368-0829, Fax +8I-66368-0829, Email kuzuya@kansai-u.ac.jp
\end{abstract}

Received: October 30, 2017 | Published: December II, 2017
Abbreviations: DNA, deoxyribonucleic acids; AAm, acrylamide; PNIPAAm, poly(N-isopropylacrylamide); PEG, polyethylene glycol; DDS, drug delivery system; HELP, highefficiency liquid phase

\section{Introduction}

Recent development of various hydrogels with unique properties is attracting scientists not only from material chemistry but also from broad research fields such as medical science. ${ }^{1,2}$ DNA is one of popular materials used to prepare hydrogels since various functional DNA motifs are already known, and easily employed to achieve intelligent hydrogels.$^{3-5}$ In addition to simple duplexes or single-stranded DNA, G-quadruplexes, unique tetrameric higher-order structures of DNA, started to be found in interesting hydrogel systems. In this mini-review, recent hydrogels utilizing G-quadruplexes are briefly explained.

\section{Discussion}

G-quadruplexes are a tetrad complexes of guanine bases associated through Hoogsteen hydrogen bonding. ${ }^{6,7}$ Since all of the 2-oxo groups with lone pairs point the central pore of the tetrads, cations, particularly $\mathrm{K}^{+}$and $\mathrm{Na}^{+}$, are strongly trapped in the center, stabilizing the quadruplex (Figure 1). It is expected that G-quadruplex structures are formed in GC-rich telomere region of chromosomes in nature, and regulating lifetime of cells by protecting the ends of chromatins.

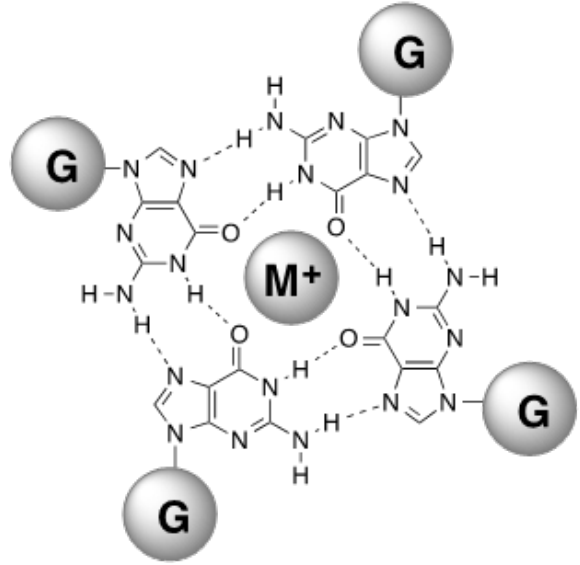

Figure I Chemical structure of a G-quartet.
The first observation of hydrogels made of G-quadruplexes formed by guanylic acids, even if the concept was not known then, had been reported more than 100 years ago. ${ }^{8}$ This finding gave rise to the first discovery of tetrameric arrangement of the guanine bases 50 years later. ${ }^{9}$ Gelation of oligo-G strands was found and has been studied well thereafter. ${ }^{10}$

Despite its known long history, hydrogels of simple guanosine and its derivatives have not often been reported in the context of materials chemistry. In 2005, Sreenivasachary and Lehn have reported gelationmediated selection of acylhydrazone G-quartets from a dynamic library of two kinds hydrazides involving guanosine-5'-hydrazide and two kinds of aldehydes. ${ }^{11}$ Quite recently, it was reported that addition of polyamines such as spermine dramatically enhances the strength of GMP hydrogels. ${ }^{12}$ Other group also reported selective attachment of dopamine-conjugated platinum (IV) to G-quadruplexes by using borate ester linkages and photocytotoxicity against cisplatin-resistant cells of them. ${ }^{13}$

The major strategy to utilize G-quadruplexes is to employ G-quadruplex-forming single-stranded DNA. Except a few examples such that Xiang et al. reported a combination of G-quadruplexforming single-stranded DNA with hydrogels formed with pure DNA junctions to achieve enzymatic cascade systems in DNA hydrogels,${ }^{14}$ or that Huang et al. utilized rolling circle amplification to obtain long single-stranded DNA forming G-quadruplexes ${ }^{15}$ most of the hydrogels involving G-quadruplexes studied to date are combinations or hybrids of synthetic polymers and single-stranded DNA. Among them, Willner's group extensively studied stimuli-responsive hydrogels utilizing G-quadruplexes. ${ }^{5,16-19}$ They first prepared acryditeDNA conjugate with relatively short DNA portion, 5-mer dAAGGG to polymerize poly-AAm bearing DNA strands. ${ }^{16}$ Addition of $\mathrm{K}^{+}$and hemin to the solution of the polymer triggered formation of hydrogels crosslinked by four-stranded hemin-G-quadruplex DNAzymes. They later conjugated G-quadruplex-forming single-stranded DNA split into halves into PNIPAAm backbone using acrydite modification, and formed hydrogels by using G-quadruplexes as cross-linking points in the presence of $\mathrm{K}^{+}$ions. ${ }^{17}$ The resulting hydrogels showed thermoresponsive phase transition between gel and solid phases. They also introduced hemin to the hydrogels to reconstitute hemin-DNAzyme that shows peroxidase activity and capable of producing polyaniline from aniline and $\mathrm{H}_{2} \mathrm{O}_{2}$ in situ. By introducing the split G-quadruplexforming strand into acrylamide backbone, instead of PNIPAAm, 
an electric sensor was constructed by layer-by-layer deposition of the DNA-acrylamide conjugate through G-quadruplex formation. ${ }^{18}$ Shape-memory hydrogels are also achievable by conjugating split G-quadruplex-forming-single-stranded DNA to AAm backbone together with duplex-forming DNA. ${ }^{19}$ G-quadruplexes and duplexes both act as cross-linkers for hydrogel formation. Introduction of $\mathrm{K}^{+}$ to the system and removal of them by 18 -crown- 6 ether reversibly trigger G-quadruplex formation and denaturation in the hydrogel, and induces its shape-change between initial shaped state and soft and shapeless state. Employment of another quadruplex structure, i-motif, realized dual-triggered system both by $\mathrm{pH}$ change and $\mathrm{K}^{+}$introduction.

Similarly, but back in 2008 , Wei et al. $^{20}$ have already used G-quadruplex-forming single-stranded DNA to crosslink poly-AAm bearing DNA strands. The G-quadruplex they employed was thrombin aptamer, and the hydrogel was used as a reversible matrix for thrombin capturing. Shao et al. ${ }^{21}$ used G-quadruplexes as $\mathrm{K}^{+}$-responsive crosslinking point of polypeptide network in combination with duplex formation between single-stranded extensions from the G-quadruplex portion and complementary strands conjugated to the polypeptides via the copper-catalyzed click reaction. Lately, Zhang et al. ${ }^{22}$ prepared molecularly imprinted PNIPAAm-AAm hydrogels containing heminG-quadruplex DNAzymes together with specific substrates TMB or ABTS, and observed enhanced enzymatic activities of the imprinted hydrogels. Zhao et al. $^{23}$ on the other hand, prepared four-stranded
hemin-G-quadruplex DNAzymes with single-stranded extensions, and used this motif as a cross-linker for AAm bearing single-stranded complementary DNA to construct a colorimetric $\mathrm{H}_{2} \mathrm{O}_{2}$ sensing system. When G-quadruplex-forming single-stranded DNA was conjugated to two acrydite groups at both ends, and used as crosslinking points of PNIPAAm hydrogel, thermo-responsive loosening of G-quadruplex structure related to PNIPAAm dehydration was observed. ${ }^{24}$

We have recently developed a new class of hydrogels utilizing G-quadruplexes without using AAm derivatives. ${ }^{25} \mathrm{~A}$ versatile hydrogel system consists of only two popular biocompatible materials; DNA and polyethylene glycol (PEG) were prepared by applying HighEfficiency Liquid Phase (HELP) synthesis of oligonucleotide, ${ }^{26}$ to overcome the scale barrier of automated solid-phase DNA syntheses. DNA-PEG-DNA triblock conjugates, in which merely four or five consecutive nucleotides are symmetrically introduced, prepared at least in gram scale with HELP, realized intelligent and biodegradable hydrogels. We named these hydrogels "DNA quadruplex gels", since they utilize reversible formation of G-quadruplexes as the crosslinking points, which is responsive to various input signals, such as $\mathrm{Na}^{+}, \mathrm{K}^{+}$, and complementary DNA strand (Figure 2). DNA quadruplex gels can be instantly prepared by the addition of various body-related fluids such as serum, artificial tear, saliva, sweat, or even cell culture media and phosphate buffered saline. They even show self-healing properties reflecting the reversible nature of G-quadruplexes.

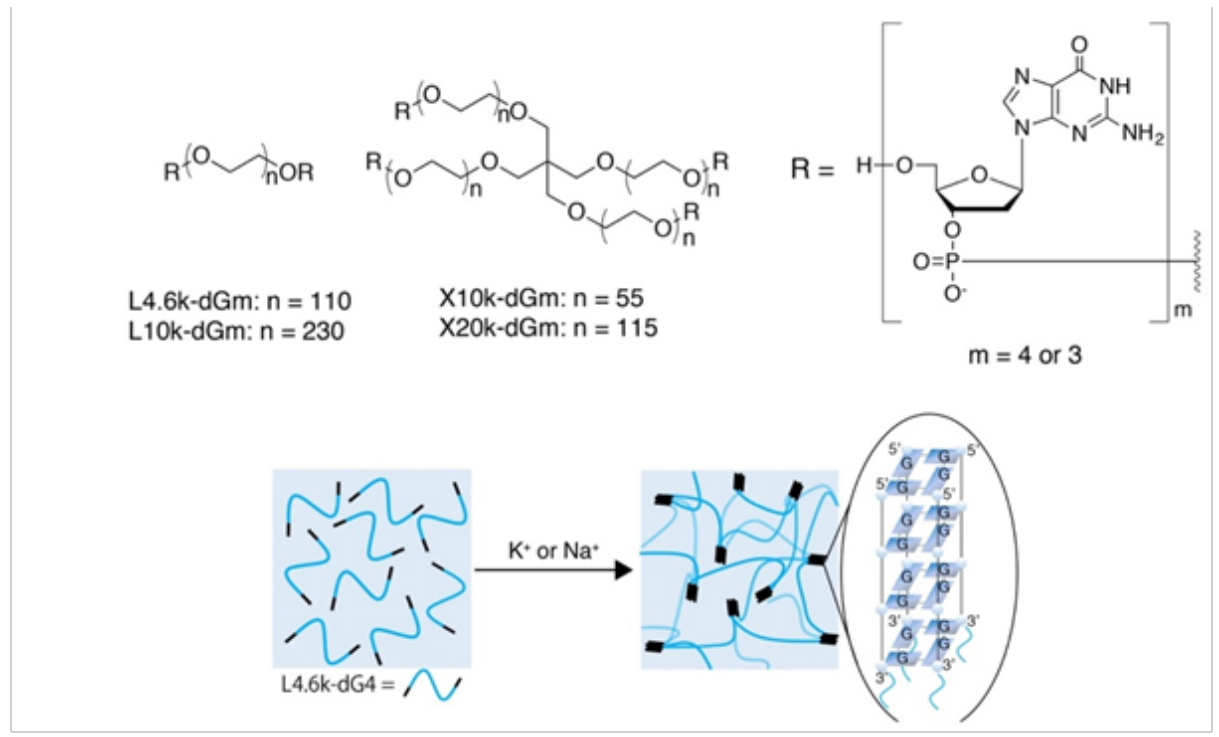

Figure 2 Schematic illustration of our DNA quadruplex hydrogels utilizing G-quadruplexes. ${ }^{25}$

\section{Conclusion}

Although most of the recent hydrogels utilizing G-quadruplexes focus on their hemin-DNAzyme activity, G-quadruplexes themselves still have many other advantageous. Quite high thermal stability compared to simple right-handed B-type DNA duplexes is a typical example. Development of still more useful hydrogel systems is quite feasible employing G-quadruplexes in various ways.

\section{Acknowledgements}

This work was supported by Precursory Research for Embryonic Science and Technology (PRESTO), "Molecular Technology and Creation of New Functions" from Japan Science and Technology Agency (JST) (JPMJPR12K4). Support from Private University Research Branding Project (2016-2021), Grant-in-Aid for Scientific Research (A) (16H01854), (B) (24350088), Grant-in-Aid for Scientific
Research on Innovative Areas "Molecular Robotics" (24104004) from the Ministry of Education, Science, Sports, Culture and Technology, Japan, and from Nihon L'Oréal are also acknowledged.

\section{Conflict of interest}

The author declares no conflicts to declare.

\section{References}

1. Ahmed EM. Hydrogel: Preparation, characterization, and applications: A review. $J$ Adv Res. 2015;6(2):105-121.

2. Hoffman AS. Hydrogels for biomedical applications. Adv Drug Delivery Rev. 2012;64:18-23.

3. Xiong $\mathrm{X}, \mathrm{Wu} \mathrm{C}$, Zhou $\mathrm{C}$, et al. Responsive DNA-based hydrogels and their applications. Macromol Rapid Commun. 2013;34(16):1271-1283. 
4. Shao Y, Jia H, Cao T, et al. Supramolecular hydrogels based on DNA self-assembly. Acc Chem Res. 2017;50(4):659-668.

5. Kahn JS, Hu Y, Willner I. Stimuli-responsive DNA-based hydrogels: from basic principles to applications. Acc Chem Res. 2017;50(4):680 690.

6. Kang $\mathrm{CH}$, Zhang X, Ratliff R, et al. Crystal structure of four-stranded oxytricha telomeric DNA. Nature. 1992;356(6365):126-131.

7. Burge S, Parkinson GN, Hazel P, et al. Quadruplex DNA: sequence, topology and structure. Nucleic Acids Res. 2006;34(19):5402-5415.

8. Bang I. Untersuchungen über die Guanylsäure. Biochem Ztschr. 1910;26:293-311.

9. Gellert M, Lipset MN, Davies DR. Helix formation by guanylic acid. Proc Nat Acad Sci USA. 1962;48:2013-2018

10. Guschlbauer W, Chantot JF, Thiele D. Four-stranded nucleic acid structures 25 years later: from guanosine gels to telomere DNA. J Biomol Struct Dyn. 1990;8(3):491-511.

11. Sreenivasachary N, Lehn JM. Gelation-driven component selection in the generation of constitutional dynamic hydrogels based on guaninequartet formation. PNAS. 2005;102(17):5938-5943.

12. Belda R, Garcia-Espana E, Morris GA, et al. Guanosine-5'monophosphate polyamine hybrid hydrogels: enhanced gel strength probed by z-spectroscopy. Chem Eur J. 2017;23(32):7755-7760.

13. Venkatesh V, Mishra NK, Romero-Canelon I, et al. Supramolecular photoactivatable anticancer hydrogels. $J$ Am Chem Soc. 2017;139(16):5656-5659.

14. Xiang B, He K, Zhu R, et al. Self-assembled DNA hydrogel based on enzymatically polymerized DNA for protein encapsulation and enzyme/ DNAzyme hybrid cascade reaction. ACS Appl Mater Interfaces. 2016;8(35):22801-22807.

15. Huang Y, Xu W, Liu G, et al. A pure DNA Hydrogel with stable catalytic ability produced by one-step rolling circle amplification. Chem Commun. 2017;53(21):3038-3041.
16. Lu CH, Qi XJ, Orbach R, et al. Switchable catalytic acrylamide hydrogels cross-linked by hemin/G-quadruplexes. Nano Lett. 2013;13(3):12981302.

17. Lu CH, Guo W, Qi XJ, et al. Hemin-G-quadruplex-crosslinked poly-Nisopropylacrylamide hydrogel: a catalytic matrix for the deposition of conductive polyaniline. Chem Sci. 2015;6(11):6659-6664.

18. Kahn JS, Trifonov A, Cecconello A, et al. Integration of Switchable DNA-based hydrogels with surfaces by the hybridization chin reaction. Nano Lett. 2015;15(11):7773-7778.

19. Lu CH, Guo W, Hu Y, et al. Multitriggered shape-memory acrylamideDNA hydrogels. J Am Chem Soc. 2015;137(50):15723-15731.

20. Wei B, Cheng I, Luo KQ, et al. Engineering target-responsive hydrogels based on aptamer-target interactions. Angew Chem Int Ed. 2008;47:331333.

21. Shao Y, Li C, Zhou X, et al. Responsive polypeptide-DNA hydrogel cross linked by G-quadruplex. Acta Chim Sin. 2015;73(8):815-818.

22. Zhang Z, Liu B, Liu J. Molecular imprinting for substrate selectivity and enhanced activity of enzyme mimics. Small. 2017;13(7):1602730.

23. Zhao H, Jiang $\mathrm{G}$, Weng J, et al. A signal-accumulating DNAzymecrosslinked hydrogel for colorimetric sensing of hydrogen peroxide. $J$ Mater Chem B. 2016;4(27):4648-4651.

24. Hasuike E, Akimoto AM, Kuroda R, et al. Reversible conformational changes in the parallel type G-quadruplex structure inside a thermoresponsive hydrogel. Chem Commun. 2017;53(21):3142-3144.

25. Tanaka S, Wakabayashi K, Fukushima K, et al. Intelligent, biodegradable and self-healing hydrogels utilizing DNA quadruplexes. Chem Asian J. 2017;12(18):2388-2392.

26. Bonora GM, Zaramella S, Veronese FM. Synthesis by high-efficiency liquid-phase (HELP) method of oligonucleotides conjugated with highmolecular weight polyethylene glycols (PEGs). Biol Proced Online. 1998;1:59-69. 